\title{
El comportamiento de la prensa durante el periodo de campañas 2018: el fortalecimiento de un discurso basado en la alternancia de Chilpancingo
}

\author{
Denia May Sánchez ${ }^{1 *}$, Lidio Edson Sánchez Rivera²
}

\section{Resumen}

Este estudio se centra en el análisis de la prensa del estado de Guerrero, específicamente, en los diarios con circulación en Chilpancingo de los Bravo, ciudad capital. En donde, de manera cuantitativa, a través de la técnica de análisis de contenido y con sustento de la teoría de la agenda-setting, se analiza la información difundida durante las campañas electorales 2018. El objetivo es analizar las características de cobertura que los diarios dieron a los tres principales candidatos que contendieron por la presidencia municipal, haciendo también una descripción, de la forma en que cada uno de ellos fue presentado durante ese periodo. Además de contrastar con los resultados de una encuesta aplicada para identificar la información que llegó al electorado durante esa etapa.

Los resultados de la investigación sugieren que los diarios con circulación en Chilpancingo, brindaron mayor relevancia noticiosa a las acciones políticas del candidato ganador, Antonio Gaspar Beltrán, quien llevó a la alternancia partidista del municipio. Por lo tanto, el discurso periodístico repercutió de manera positiva en la figura del candidato ante la opinión pública.

\section{Abstract}

This study focuses on the analysis of the press in the state of Guerrero, specifically, on the newspapers with circulation in the capital city. Where, quantitative, and through the content analysis technique and drawing on the "agenda-setting" theory, the information disseminated during the 2018 election campaign was analyzed. The aim of this work is getting to know the coverage that the newspapers assigned to the three main political candidates that contended for the municipal presidency, also making a detailed description on how each one of them was exposed during that period. Furthermore, the results were compared with a survey applied to identify the information that reached the voters on that stage.

The research's findings suggest that local newspapers gave the most relevant press' coverage to the winning contender's political actions, Antonio Gaspar Beltrán, who led the municipality to a political alternation. Therefore, the journalistic speech positively affected the candidate's image in terms of public opinion.

\section{Palabras Clave}

Análisis de discurso, Campañas electorales, Prensa, Agenda setting, Alternancia.

\section{Keywords}

Political discourse analysis, Election campaigns, Press, Agenda-setting, Political alternation.

${ }^{1}$ Maestra en Comunicación y Relaciones Públicas con Licenciatura en Ciencias de la Comunicación por la Universidad Autónoma de Guerrero. ${ }^{2}$ Maestro en Comunicación y Relaciones Públicas con Licenciatura en Ciencias de la Comunicación por la Universidad Autónoma de Guerrero. *Autor para correspondencia: deniamay@gmail.com

\section{Introducción}

En el año 2018, se efectuó uno de los procesos electorales más complejos que haya ocurrido en la historia de los
Estados Unidos Mexicanos, el cual tuvo resultados que evidenciaron los cambios en el escenario político de todo el país, impactando a partidos y candidatos. Chilpancingo, 
capital del estado de Guerrero, no fue la excepción. Siendo una ciudad con historia y características partidistas de derecha, se enfrentó a un cambio radical con los resultados obtenidos, puesto que favorecieron al candidato representante de izquierda, dejando atrás "la ventaja electoral que se dio por 86 años al Partido Revolucionario Institucional (PRI)” en las elecciones municipales (De Dios Palma, 2018).

Los resultados de las elecciones llevadas a cabo el 1 de julio de 2018, tanto en el orden federal como en el local, mostraron el interés de la ciudadanía por un cambio que contribuyera a la mejora y el bienestar social. Por lo tanto, la labor informativa de la prensa local jugó un papel importante en esta contienda electoral, ya que fungió como uno de los principales canales por el cual la población pudo conocer las acciones y propuestas de los candidatos.

Durante todo el proceso, se suscitaron movilizaciones y eventos en la ciudad, en los cuales, la cobertura periodística fue primordial para la estructuración del discurso de los candidatos que contendieron por la alcaldía. Es así como cada uno de ellos articuló y pronunció su propio discurso basado en las características de su partido, sus propuestas, el contexto y la opinión pública. Tales elementos, se volvieron necesarios a la hora de analizar la forma y el contenido de los mensajes difundidos en la prensa.

La contienda fue compleja debido a tres situaciones que enmarcaron el contexto: la primera fue el fenómeno provocado por el partido Movimiento de Regeneración Nacional (Morena), abanderado por el entonces candidato a la presidencia de la república, Andrés Manuel López Obrador, quien a través de su influencia, ganó votos a favor de su partido. La segunda situación que se hizo presente fue el arraigo de la población guerrerense por el Partido Revolucionario Institucional (PRI); el cual a nivel estatal permaneció hasta el año 2005, recuperando el puesto en el 2015 con el actual gobernador Héctor Astudillo Flores. Asimismo, en la presidencia municipal, el partido estuvo en la preferencia del pueblo de manera ininterrumpida por varias décadas. La tercera circunstancia referida, es sobre el despegue del Partido de la Revolución Democrática (PRD) en la ciudad capitalina, con Antonio Gaspar Beltrán como candidato al frente, quien resultó ganador de estas elecciones. Por ello, es importante estudiar el comportamiento de la prensa en este periodo, pues se trata de un momento crucial en la vida política y democrática del país y de cada uno de sus órdenes de gobierno, tomando en cuenta que "A partir de procesos de selección de algunos aspectos de la realidad, los medios resaltan permanentemente estos atributos contribuyendo a construir determinadas percepciones sobre los candidatos, sus acciones y sus propuestas." (Acevedo y Macassi, 2016, p. 175).

El objetivo de esta investigación es describir el comportamiento de la prensa durante el periodo de campañas 2018, en su labor de difundir el discurso y las acciones de campaña de los principales candidatos a la presidencia municipal de Chilpancingo. Usando un método descriptivo cuantitativo, a través del análisis de contenido y complementando con una encuesta, para así, contrastar la información resultante de ambas técnicas. De esta manera: se conocerá la relevancia noticiosa que se dio a la información difundida sobre los tres candidatos; se analizará el discurso periodístico a través de los temas y titulares; y se conocerán algunas percepciones de ciudadanos lectores que se mantuvieron informados durante el proceso electoral, a través de la prensa:

Conocer el perfil discursivo de la comunicación política permite entender la cultura política desde el ángulo del comportamiento de quién comunica y qué comunica, y el canal o medio que le sirvió de primer intermediario; igualmente a quién se comunica y con qué efectos y en qué contexto. (Cotteret, 1977, citado en Ascanio, 2014, p. 17)

\section{Marco teórico-referencial}

Actualmente, se considera necesario el monitoreo de los medios de comunicación para llevar un control de los mensajes que se emiten a diario, sobre los temas relacionados con la política o las cuestiones electorales. Esto se implementó de manera oficial a partir de las reformas del 2007 y 2008, debido a la participación e influencia de los medios en esa clase de procesos. Por lo tanto, los actores políticos tienen que considerar la actividad mediática para imponer su discurso, puesto que son una herramienta crucial para lograr sus objetivos. Quienes tengan esa capacidad de imponer su discurso, tendrán mayores posibilidades de apoyo "sumar adeptos y conquistar votos que les permiten implementar sus 
estrategias políticas. En otras palabras, el discurso político demarca el terreno de lo políticamente factible en momentos y contextos sociales determinantes" (Welsch, 1983, en Ascanio, 2014, p. 7-8).

El análisis del discurso político se vuelve complejo porque involucra las acciones de los actores políticos, las cuales representan solo una parte, mientras que el contexto pasa a ser algo más significativo. Por ello dicen que el eje central del contexto se verá reflejado en los participantes y sus acciones "pero podríamos ir más lejos analizando los contextos ampliamente desde el punto de vista de los sucesos políticos y comunicativos, con sus escenarios propios (tiempo, lugar, circunstancias), ocasiones, intenciones, funciones, metas, e implicaciones políticas o legales" (Van Dijk y Mendizábal, 1999, p. 15).

El discurso cumple diversas funciones que pueden ayudar o hacer caer la imagen de los candidatos, esto a través de los medios de comunicación, de tal modo que esa influencia genera una visión sobre los actores políticos o gobernantes, así pues "no solo comunica algo, sino que además construye, mejora o deteriora la imagen del político y permitirá conservar -o no- su popularidad cuando se encuentre en el ejercicio de una responsabilidad pública" (Meyenberg y Lugo, 2011, p. 18).

En materia electoral, el candidato o el partido tienen que planificar una estrategia capaz de captar votos, coherente con el contexto y que tenga la facultad de dirigir y persuadir a las masas. Una correcta imagen política tiene la capacidad de transformar y dirigir a la opinión publica. Por ejemplo, Martínez Pandiani (2004) hace distinguir que la imagen política debe ser un agregado entre las percepciones referidas de diversos aspectos del ser y actuar, incluso al realizar acciones y llevar a cabo propuestas, para que coincidan con la imagen que se desea proyectar.

La importancia de una imagen política es la posibilidad de influir en las relaciones emocionales del votante con los candidatos, por eso se requiere que el aspirante al cargo coincida con la realidad de la ciudadanía. Sin embargo, para García, D’Adamo y Slayinzky (2005), la información que habla del candidato no siempre es positiva, dado que, a lo largo de una gran variedad de escenarios, eventos y personas, las experiencias positivas se han encontrado como menos influyentes en la formación de juicios que las negativas, o aspectos negativos de los estímulos. Por ende, en algunos casos, en los juicios de la opinión pública, las debilidades en la imagen de cualquier candidato se vean más importantes que las fortalezas.

\section{La política, los medios y la agenda}

Martínez Pandiani (2004) señala que "los medios masivos se transforman en la usina de información política más consultada por los ciudadanos al momento de tomar decisiones electorales" (p. 48). Cabe mencionar que el papel de los medios impresos sigue siendo relevante en el proceso político, puesto que la importancia de la televisión y la radio, radica en que sus audiencias son mucho mayores que la de los medios impresos, pero para el establecimiento de la agenda, los medios impresos son muy influyentes, a tal grado de ser los que crean el debate público que se trasladará a la televisión. Como señala Muñoz (2003), "vivimos bajo el imperialismo de la televisión, pero el medio rey sigue siendo el periódico" (p. 13).

Sweeney (1995) hace referencia a las campañas políticas como un ejercicio de comunicación entre los aspirantes a un cargo y una audiencia de votantes, y es que, actualmente las campañas son hechas con el fin de convencer al electorado a través de los medios masivos de comunicación. Asimismo, retomando lo que dice Aguayo (1998): “Los medios de información tienen una importancia estratégica para la vida política: pueden ser una columna que soporte la democracia o un instrumento utilizado por el gobierno autoritario para controlar las ideas que llegan a la población" (p. 274).

Larry Sabato (1981) hace hincapié en que hoy en día es común que en todos los procesos electorales se enfrenten a un creciente desalineamiento político de los votantes. Ya no se pueden crear sistemas mediáticos sencillos, como lo explicaba Campbell (1960), donde su "atajo cognitivo" creaba una toma de decisiones sin contar con toda la información, pues era el alineamiento con un partido el que señalaba el comportamiento electoral. Es decir, para conocer el proceso de toma de decisión de los votantes, no solo hay que conocer su alineamiento partidario, sino que se tienen que buscar las ideas, los motivos, sentimientos y propuestas con las que simpaticen y los hagan reaccionar al "voto" o al "no voto".

Los votantes de hoy, han evolucionado respecto a las variables usuales para efectuar su derecho al voto. Nie, Verba 
y Petrocik (1976) han conferido más importancia a los issues como las decisiones del gobierno en turno, la marcha de la economía o a la valoración de los diferentes líderes. En cuanto a esto, se puede entender desde el punto de vista político de Santillán (2016) que "el concepto issues hace referencia a una cuestión pública que involucra un conflicto político y social, que es presentado y discutido en los medios de comunicación para la toma de soluciones políticas" (p. 191).

La teoría del establecimiento de la agenda o agenda setting según, McCombs y Shaw (1972), estudia la participación de los medios para establecer una agenda con temas anticipados sobre aquello que publicarán, dando relevancia o contrarrestando la importancia de los hechos a ámbito nacional e internacional, con el propósito de aumentar su audiencia, generar un impacto social y mantener un control de la información. Dicha selección temática que hacen los medios, es la que tiene cierta influencia en el público, y no de manera contraria. El establecimiento de la agenda se desglosa de la teoría de los efectos del autor Paul Lazarsfeld (Toussaint, 2004), que proponía que la influencia de los medios es indirecta y está filtrada por la capacidad cognitiva de cada ser humano. Con este enfoque se cuestionan los planteamientos sobre el poder que tienen los medios dentro de la sociedad. La agenda setting se encarga de asignarles un papel importante a los medios informativos a la hora de dar marcha a las estrategias establecidas en una campaña. En este proceso, surge la prominencia de objetos y atributos en la opinión pública (Berkowitz y Rogers, 1986).

Es también importante hacer referencia a la "relevancia", definida como la forma en que la información será visualizada a través de características como frecuencia de cobertura, tamaño, ubicación o disposición respecto a otro tipo de información (Muñiz, et al., 2016). De esta manera, McCombs (2006) también destaca que su interés se mantiene debido a la idea de que, en análisis de relevancia mediática, las noticias publicadas en las portadas de los diarios, generan al menos el doble de lectores, esto en comparación con las que son publicadas en el interior. Teniendo así, tres dimensiones en la relevancia de la agenda mediática: la atención, la prominencia y la valencia. La valencia remite a la dimensión afectiva, tomando en cuenta a las "emociones" que acompañan al mensaje. Como han afirmado Kiousis y Shields (2008), aunque la literatura académica ha identificado diversos tipos de materiales como los discursos, la publicidad política y las conferencias de prensa, la mayor parte de la investigación empírica se ha centrado en las notas de prensa.

\section{Contexto de las elecciones 2018 en Chilpancingo}

En Chilpancingo, el PRI se mantuvo como el representante de derecha por más de ocho décadas, por tanto, también se mantuvo en el poder durante este tiempo. Sin embargo, en el 2018, la candidata representante de este partido, Beatriz Vélez Núñez, se enfrentó a una contienda muy diferente en la cual no logró vencer a sus oponentes. Cabe mencionar que, en el caso del partido Morena, existió mucha proliferación con el electorado gracias a su estrategia en conjunto con la imagen del candidato a la presidencia de la república mexicana, Andrés Manuel López Obrador, popular candidato que ganó el puesto de presidente de la republica con más de un $60 \%$ de votos. Por tal razón, es necesario conocer la configuración del discurso que utilizó el candidato de la “Coalición Por Guerrero al Frente” (PRD-PAN-MC), Antonio Gaspar Beltrán, ganador de las elecciones a la presidencia municipal de la capital del estado de Guerrero, quien el día cinco de julio recibió la constancia de mayoría en el distrito local 02 del Instituto Electoral y de Participación Ciudadana del Estado (IEPC). Ahí se detalló su triunfo con 42 mil 541 votos, mientras que la candidata de Morena, Silvia Alemán Mundo, alcanzó los 37 mil 227 votos y la candidata de la coalición PRI-PVEM, Beatriz Vélez Núñez, logró 23 mil 503 sufragios.

\section{Metodología}

Para esta investigación se utilizó un modelo metodológico cuantitativo y descriptivo, en donde fue necesario utilizar la técnica de análisis de contenido y se complementó con una encuesta, con la finalidad de conocer el comportamiento de la prensa respecto a los candidatos en campaña y también algunas características que percibió el público sobre ellos. Se contó con el registro de información proporcionado por el Centro Estatal de Monitoreo de Medios del estado de Guerrero, analizando los distintos documentos hemerográficos que fueron recolectados durante las campañas electorales, mismas que definieron la agenda de los medios locales.

La pregunta de investigación que dio dirección a este trabajo se presenta a continuación: ¿cuáles fueron las 
características de cobertura que los diarios dieron a las campañas de los tres principales candidatos que contendieron por la presidencia municipal de Chilpancingo en las elecciones 2018 ?

En este caso, el análisis de contenido funge como una técnica prescindible para poder detectar la información que brinde lo necesario para interpretar los mensajes.

El corpus analizado comprende la información de once diarios: Vértice, Diario 17, El Sol de Chilpancingo, Pueblo, El Sur, Redes del sur, Novedades Acapulco, El sol de Acapulco, La Jornada Guerrero, Diario 21 y Diario de Guerrero, teniendo un total de 449 ejemplares analizados durante el periodo de campañas por el ayuntamiento de Chilpancingo. Este inició el sábado 19 de mayo y terminó el miércoles 27 de junio de 2018. La consulta hemerográfica corresponde al registro de las notas diarias en donde hubo mención de los tres principales candidatos.

Es importante indicar que los diarios se seleccionaron por su cobertura, mayor lectoría y su presencia en el municipio de Chilpancingo de los Bravo. Retomados del catálogo de medios impresos del periodo ordinario 2018 que fue aprobado por el Instituto Electoral de Participación Ciudadana del estado de Guerrero (IEPC Gro).

Con este análisis se trata de extraer y procesar datos que aluden a la relevancia y a las condiciones mismas en que se produjeron las notas informativas, tanto de estructura como cognitivas. Tales datos como: la valoración que se tiene en cada nota (positiva, negativa, neutral) de cada candidato; el tamaño que ocuparon las notas en las planas (una plana, media plana un cuarto de plana, un octavo de plana); la cantidad de menciones durante la campaña (de los tres candidatos); el género periodístico de cada mención (nota informativa, opinión y análisis, entrevista, fotonota, crónica o reportaje). Además, para profundizar, se toma en cuenta a los titulares y las temáticas de las piezas informativas, llevando un análisis puntual y descriptivo de la forma en que se elaboró el discurso a través de la prensa de cada uno de los candidatos señalados. Generando una línea temporal de los acontecimientos ocurridos en cada una de las semanas que conformaron el periodo de campañas y detectando las principales palabras utilizadas para la redacción de las notas presentadas.
La encuesta se aplicó a ciudadanos que votaron en las elecciones 2018 en la ciudad capital. Cabe mencionar que Chilpancingo es la ciudad del estado de Guerrero en donde se encuentra el mayor número de personas registradas en el padrón electoral, por lo tanto, se tomó una muestra de ese total.

\section{Resultados}

\section{Análisis de contenido}

Cantidad de menciones. Con este estudio, se logró conocer que Antonio Gaspar Beltrán, fue mencionado en 226 ocasiones durante todo el periodo de campañas, la candidata Beatriz Vélez lo seguía con 184, dejando muy por debajo a la candidata Silvia Alemán con 39 menciones. Aunque había una diferencia marcada en la cantidad de notas de los dos primeros candidatos -Antonio y Beatriz-, se encontró un patrón de frecuencia similar en el periodo analizado. En la Figura 1, se puede apreciar el ritmo de la presencia en prensa de estos dos candidatos. La cantidad de menciones sube y baja cada semana casi a la par.

Figura 1. Menciones por semana.

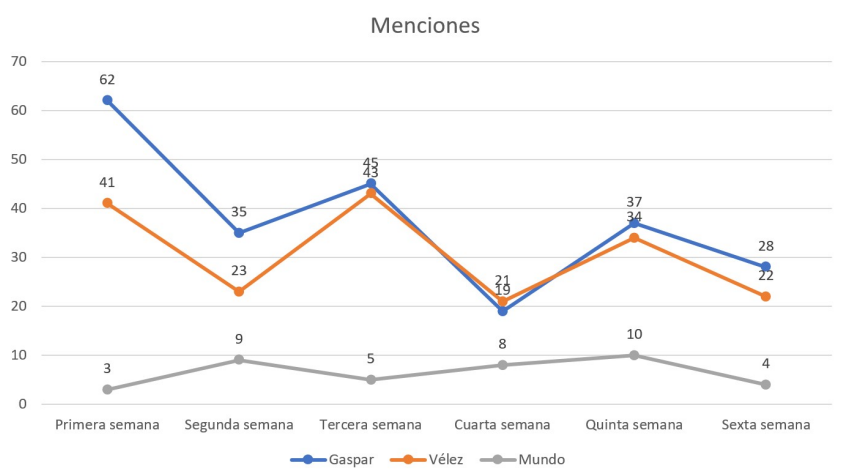

Nota: elaboración propia, con base en la información recuperada del Centro Estatal de Monitoreo de Medios Guerrero.

Espacio en diarios. Las medidas consideradas para determinar el espacio brindado a los candidatos, fueron cinco: una plana, tres cuartos de plana, media plana, un cuarto de plana y un octavo de plana. Así, en el tamaño de las piezas informativas en donde se mencionaron a los candidatos, destaca el de $\frac{1}{4}$ de plana que fue utilizado 263 veces $(58.6 \%)$, mientras que el espacio de plana entera solo se ocupó una 
vez a lo largo de este periodo, con el título "La disputa por Chilpancingo y Acapulco", en el Diario 17 del día 4 de junio, haciendo alusión al candidato Antonio Gaspar. Se trató de una nota de opinión y análisis que fue publicada durante la tercera semana de campañas, abordando los recorridos y compromisos del candidato, con comentarios como el siguiente: "Toño Gaspar como popularmente lo conocen en los barrios, colonias y comunidades, escucha diariamente las inconformidades de la gente, establece compromisos y se deja apapachar".

El tamaño $\frac{3}{4}$ de plana se convirtió en el menos frecuente (17 veces) para mencionar a todos los candidatos, abarcando el $3.8 \%$ del total de publicaciones durante las campañas. En este tamaño es importante destacar que la candidata Silvia Alemán solo tuvo una mención, y fue debido a comentarios hechos por el candidato Antonio Gaspar, lo que se transcribió con las propias palabras del contendiente y no las del reportero. En cuanto al tamaño de $\frac{1}{2}$ plana, Gaspar y Vélez tuvieron una diferencia de dos en su uso; Vélez fue quien más ocupó este tamaño con 53 repeticiones y Gaspar la siguió con 51. Cabe mencionar que el tamaño de $\frac{1}{4}$ de plana fue en el que más se repitió Silvia Alemán, con 33 apariciones de las 39 en toda su campaña. Mientras que el tamaño de $\frac{1}{8}$ de plana fue usado 60 veces (13.4\% del total): 40 para mencionar al candidato Antonio Gaspar, 19 para Vélez y una para Silvia Alemán.

Menciones clasificadas por género periodístico. La nota informativa, caracterizada por tener una información objetiva y directa, fue la más utilizada durante las campañas con un $83.5 \%$. En dicho género, Gaspar tuvo 187 menciones, Vélez 149 y Alemán 39.

En el género de opinión y análisis, la suma de menciones de Gaspar y Vélez fue de 23, de las cuales, en la primera semana, 11 fueron para él y ocho para ella. El género de entrevista fue usado cinco veces durante las primeras dos semanas de la campaña con Gaspar como protagonista. Mientras que Vélez fue entrevistada tres veces: en la tercera, cuarta y quinta semana. La fotonota fue utilizada para referirse únicamente a los candidatos Gaspar y Vélez, quienes ocuparon ocho y nueve alusiones respectivamente. Siendo la última semana donde más veces los mencionaron en este género con tres para Gaspar y cuatro para Vélez.

El recurso de crónica fue utilizado solo una vez en el periodo de campañas, en el periódico Vértice, el día 8 de junio, con el título "En el mercado se vuelcan en apoyo a Toño Gaspar", pieza informativa en la cual se mencionó el recorrido del candidato por el mercado R. Leyva Mancilla (mercado principal de la Ciudad de Chilpancingo) y ocupó un espacio de $\frac{1}{2}$ plana. Respecto al reportaje, solo se utilizó dos veces en todo el periodo el día 23 de junio y en ambos casos se hizo referencia al candidato Gaspar, presentándolo con valoración positiva: en el diario Vértice con un cuarto de plana, el título fue "La capital. Propone Toño Gaspar unificar Chilpancingo"; y en el Diario de Guerrero con media plana, el reportaje se tituló “Contexto Político, Toño Gaspar: En la ruta del Triunfo".

Menciones según su valoración. Conforme a la valoración obtenida por los candidatos en todas sus menciones, resaltan 232 positivas y 186 neutrales, las cuales suman el $93 \%$, siendo el $7 \%$ restante, 31 notas con valoración negativa. Véase Tabla 1.

Antonio Gaspar y Beatriz Vélez fueron los más referenciados con valoraciones positivas, con 124 y 94 menciones, respectivamente. Mientras que Silvia tuvo solamente 14. Durante el periodo de campañas, esta última candidata no fue mencionada en notas valoradas como negativas, mientras que Gaspar estuvo en 12 y Vélez en 19. Cabe señalar que la candidata Vélez tuvo siete valoraciones negativas más que el candidato Gaspar. En la primera semana de campaña, Antonio Gaspar alcanzó las 9 valoraciones negativas y Beatriz tuvo seis, para que en la segunda semana Antonio bajara a solo una valoración negativa, mientras Beatriz alcanzó las 11 menciones negativas. Respecto a las menciones neutrales, Gaspar obtuvo 90, Vélez 71 y Alemán 25. Convirtiéndose la valoración neutral, la más frecuente durante la campaña para la candidata Silvia Alemán Mundo.

\section{Línea temporal}

La línea de tiempo realizada abarca el periodo comprendido entre el 19 de mayo y el 27 de junio de 2018, días en que se realizaron las campañas por el ayuntamiento municipal de Chilpancingo. Esto con la finalidad de poder llevar un orden en las notas más relevantes para la investigación. La línea fue dividida en seis partes que representa cada una de ellas a una semana. Véase Figura 2. 
Tabla 1. Menciones contadas por su valencia/valoración.

\begin{tabular}{lllll}
\hline \multicolumn{1}{c}{ Candidato } & \multicolumn{1}{c}{ Positivas } & \multicolumn{1}{c}{ Negativas } & \multicolumn{1}{c}{ Neutrales } \\
\hline Gaspar & 124 & 12 & 90 & 71 \\
\hline Vélez & 94 & 19 & 25 \\
\hline Alemán & 14 & 0 & 186 \\
\hline TOTAL & 232 & 31 &
\end{tabular}

Nota: elaboración propia, con base en la información recuperada del Centro Estatal de Monitoreo de Medios Guerrero.

Figura 2. Línea temporal sobre menciones relevantes durante las campañas electorales 2018.

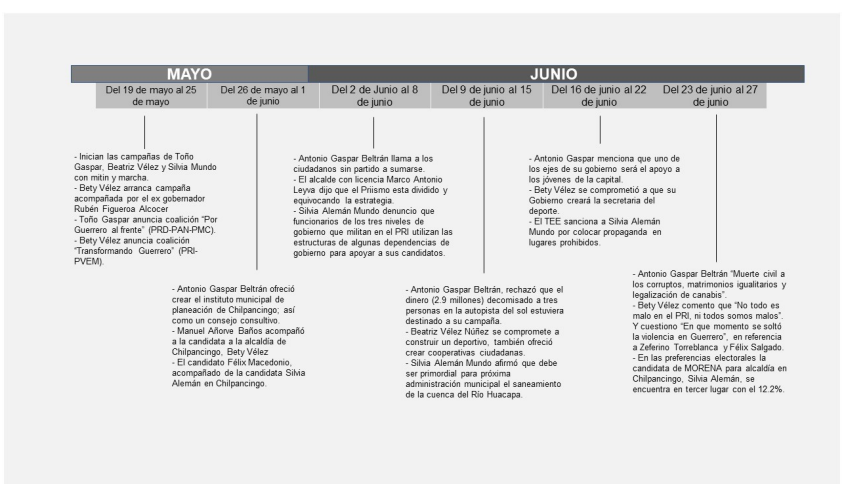

Nota: elaboración propia, con base en la información recuperada del Centro Estatal de Monitoreo de Medios Guerrero.

Con esta división se pudieron correlacionar los hechos más relevantes de cada semana, en donde tuvieron presencia los candidatos.

\section{Primera semana. Del sábado 19 de mayo al viernes 25 de mayo del 2018}

Se observaron algunos hechos importantes, típicos de una campaña política. El primero fue que, los tres candidatos dieron inicio a sus campañas en la ciudad de Chilpancingo, organizaron un mitin político con sus respectivos seguidores, además de realizar una marcha por las principales calles de la ciudad en donde invitaron a más personas a unirse a su proyecto. Por su parte Beatriz Vélez, en sus recorridos por la ciudad se hizo acompañar por actores políticos de su partido, tales como el presidente nacional del PRI, René Juárez Cisneros y el ex gobernador Rubén Figueroa Alcocer, personajes de relevancia en el ámbito político.

Otro hecho relevante fue que los candidatos Antonio Gaspar y Beatriz Vélez, anunciaron que sus partidos participarían en coalición con otros. Quedando así, el primero con la coalición PRD-PAN-PMC, donde los presidentes estatales de cada uno de estos partidos dieron el apoyo al candidato; la candidata Beatriz Vélez, con la coalición PRI-PVEM. En esta primera semana se observó que las propuestas de los candidatos fueron similares, ya que se inclinaban por el mejoramiento de los servicios públicos y la seguridad, así como la eliminación de la corrupción.

En cantidad de menciones en los periódicos, el candidato Antonio Gaspar obtuvo el mayor número con 62 notas, 16 notas más que la suma de sus dos contrincantes. Esta cantidad se representa como el $27.5 \%$ de menciones alcanzadas durante toda su campaña. En contraste, la candidata Silvia Alemán solo fue mencionada tres veces, convirtiéndose esta semana en la que obtuvo menos menciones en toda la campaña.

\section{Segunda semana. Del sábado 26 de mayo al viernes 01 de junio del 2018}

La mayoría de propuestas incluyó los servicios públicos y sus mejoras. Antonio Gaspar Beltrán informó que si ganaba, crearía el Instituto Municipal de Planeación de Chilpancingo además de un Consejo Consultivo. Con estos anuncios fortaleció su estrategia e imagen al acercarse y escuchar a la gente inconforme, dejándose ver como el hombre que escucha de primera mano y se preocupa por la ciudadanía. Además, agregó que en su gobierno todos los proyectos y decisiones se tomarían con respaldo de la razón social. Mientras que la candidata priista, Beatriz Vélez, seguía en sus recorridos por las colonias y barrios de la ciudad, acompañada por actores políticos de su partido. La candidata mencionó que en su cargo anterior como diputada federal, fue la única persona que logró una partida de al menos diez millones de pesos para remodelar la Avenida Álvarez, una de las más importantes de la ciudad. Con esas palabras dio a entender a la ciudadanía que solo 
ella estaba capacitada para obtener recursos del erario federal. Esta mención de Beatriz Vélez contrasta con las propuestas del consejo consultivo de Antonio Gaspar, luego de que el candidato indicó que la remodelación hecha a la Avenida Álvarez no tenía una clara razón social.

En esta semana, Silvia Alemán tuvo nueve menciones, es decir, el $23 \%$ del total. Dos notas pertenecientes al día miércoles 30 de mayo, abordaron las declaraciones que hizo sobre el supuesto apoyo por parte de la Universidad Autónoma de Guerrero y otras instituciones, a los otros candidatos, poniendo en controversia el uso indebido de instituciones de gobierno para beneficio de sus oponentes.

Otras seis notas de ese mismo día trataron sobre la respuesta de la UAGro (rector: Javier Saldaña Almazán), sobre dichas acusaciones: "Rechazan y lamentan en la UAGro acusaciones de candidata de Morena" (Diario 17); "Rechaza la UAGro, acusaciones de la candidata de Morena a la alcaldía" (Diario de Guerrero); "La UAGro rechaza intromisión en el proceso electoral" (El Sol de Chilpancingo); "Pide UAGro a candidatos que respeten la autonomía universitaria" (Pueblo); "Rechaza la UAGro acusación de favorecer a candidatos del PRI" (Diario 21) y; "Está prohibido el proselitismo dentro de la UAG, responde Rectoría a Silvia Alemán” (El sur).

\section{Tercera semana. Del sábado 02 de junio al viernes 08 de junio del 2018}

La estrategia de Antonio Gaspar Beltrán se centró en hablar de la alternancia; invitar a candidatos de otros partidos; recibir a ciudadanos reconocidos con antecedentes priistas que se sumaron a su proyecto; y recorrer las colonias de la ciudad. Los titulares de las notas se reforzaron con testimonios de ciudadanos, además, la información alude principalmente a los eventos del candidato, los cuales fueron programados en sus redes sociales.

El PRI se vio afectado en el ámbito local, ya que el presidente municipal priista con licencia, Marco Antonio Leyva Mena, afirmó que su partido estaba dividido internamente, destacando que esa era la razón por la cual los candidatos aspirantes a un cargo político, en las elecciones municipales, no pudieran crear estrategias sólidas y unificadas. Con estas palabras, el PRI y sus candidatos, sufrieron un daño en su imagen política, puesto que el contexto en que se mencionó, hizo obviar el caos y los problemas internos que se estaban gestando. Mientras tanto, Silvia Alemán Mundo denunció que el alcalde priista Jesús Tejeda Vargas, junto con su partido, estaban interviniendo en contra de la visita que haría el entonces candidato a la presidencia nacional, Andrés Manuel López Obrador. Tal visita, buscaba que se demostrara la influencia del partido Morena sobre sus seguidores en la ciudad de Chilpancingo, a la vez que reforzaría la unión de la coalición "Juntos haremos historia" (Morena-PT-PES). En las menciones de prensa escrita, Silvia Alemán Mundo siguió con su estrategia de apostar a las propuestas sobre el mejoramiento de los servicios públicos y la lucha contra la corrupción en la ciudad. También denunció los actos "antiéticos" de sus contrincantes.

\section{Cuarta semana. Del sábado 09 de junio al viernes 15 de junio del 2018}

En este lapso, los tres candidatos sumaron 48 menciones, siendo la menor cantidad durante toda la campaña, también representó el mínimo de menciones en la campaña de Antonio Gaspar (19 menciones) y de Beatriz Vélez (21 menciones). Mientras que Silvia Alemán se mantuvo en ocho menciones, representando para ella, el $20.5 \%$ de las menciones de toda su campaña.

Tras ser acusado Antonio Gaspar de obtener dinero ilegítimo para financiar su campaña, los diarios desmintieron este evento durante dos días consecutivos, usando 10 notas de las 19 ocupadas en la semana, publicadas el 11 y 12 de junio (lunes y martes) para ya no volver a mencionar el tema posteriormente. El resto de las notas, nueve, se basó en mostrar el apoyo a las comunidades y un acercamiento a la iglesia católica. A la par de esto, Beatriz Vélez también desmintió, el 12 de junio, la existencia de un desabasto del cuadro básico de medicamentos en centros de salud de comunidades y colonias de la capital del estado, puesto que previo a las elecciones, la candidata fungió como secretaria general de la sección 36 del Sindicato Nacional de los Trabajadores de la Secretaría de Salud (SNTSA), tal situación se vio reflejada solo en una de las 21 notas que se publicaron en esa semana. Beatriz Vélez, tras seguir con la propuesta de mejorar servicios públicos y pelear contra la corrupción, agregó a sus propuestas, el compromiso de construir un deportivo para los jóvenes y crear cooperativas ciudadanas, siendo esto mencionado en seis notas. Mientras que Silvia Alemán en las ocho piezas 
informativas que tuvo en la semana, hizo hincapié sobre la necesidad y urgencia del saneamiento de la cuenca del Río Huacapa.

\section{Quinta semana. Del sábado 16 de junio al viernes 22 de junio del 2018}

Antonio Gaspar Beltrán concluyó con sus recorridos en calles y colonias, anunciando que su campaña sería permanente. También agradeció la adhesión del Partido Impulso Humanista y se le mencionó en la apertura de un torneo de futbol infantil. La candidata Beatriz Vélez, siguió en campaña señalando el retraso en los servicios de la ciudad y asegurando que ella entendía la situación debido a su origen humilde. Además, afirmó su compromiso con los grupos juveniles en la creación de la secretaria del deporte.

En cambio, Silvia Alemán, obtuvo diez menciones, el $25.6 \%$ en toda su campaña, de las cuales cuatro se valoran como negativas: una debida a la sanción impuesta por el Tribunal Electoral del Estado de Guerrero, por la amonestación pública al colocar propaganda en lugares prohibidos; y tres menciones más se debieron a las alusiones por parte del candidato Antonio Gaspar, quien aseguró que Silvia Alemán se encontraba por debajo de él en cuestión de votos. En las notas positivas destaca una mención que indica la adición de "panistas, perredistas y grupos de jóvenes al coincidir que es la mejor propuesta para cambiar al municipio de Chilpancingo".

\section{Sexta semana. Del sábado 23 de junio al miércoles 27 de junio del 2018}

El candidato Antonio Gaspar se reunió con dirigentes de su coalición, líderes sociales y grupos juveniles, quienes mencionaron "muerte civil a los corruptos, matrimonios igualitarios y legalización de cannabis" (Diario de Guerrero, 23 de junio). Además, advirtió la toma de precauciones con el PRI, ya que presuntamente compraría votos en las elecciones. Beatriz Vélez, siguió con la estrategia de acompañar a eventos a los otros candidatos de su partido, así como visitar colonias y barrios. También mencionó que no todos los candidatos de su partido eran iguales, haciendo referencia a los malos gobiernos que recientemente hubo por parte de su partido. La candidata Silvia Alemán apareció en cuatro notas, de las cuales, tres mencionaban que estaba en tercer lugar ante sus contrincantes, pues para entonces, tan solo alcanzaba el $12.2 \%$ de popularidad en las encuestas realizadas.

\section{Palabras más recurrentes en los titulares}

Las veinte palabras con mayor repetición en las notas del candidato Antonio Gaspar Beltrán, fueron las siguientes: coalición (85), campaña (42), capital (41), cambio (29), gente (22), comunidades (20), alternancia (18), colonias (18), ciudad (15), recorrido (14), denunció (13), dinero (12), pueblo (12), líder (11), proyecto (11), pueblos (11), sierra (11), afirmó (10), ciudadanos (10), dirigentes (10).

Beatriz Vélez Núñez: capital (36), coalición (31), campaña (30), colonias (25), gente (23), comunidades (22), distrito (19), ayuntamiento (18), compromiso (18), federal (18), mujer (18), propuesta (16), ganar (15), afirmó (14), familias (14), respaldo (14), acompañada (13), recursos (13), administración (12), apoyo (12).

Esto muestra que los candidatos Antonio Gaspar y Beatriz Vélez, coinciden con siete palabras: afirmó, campaña, capital, coalición, colonias, comunidades y gente. Seis de esas palabras se encontraron en las diez más utilizadas en los titulares de los diarios analizados.

Silvia Alemán Mundo: aseguró (10), favor (10), administración (8), electoral (8), Huacapa (8), primordial (8), próxima (8), río (8), saneamiento (8), acusó (6), encuentra (6), involucrada (6), proceso (6), rechazó (6), votos (6), caso (4), resolver (4), resultados (4), acompañada (3), capital (3). Alemán solo coincidió dos veces con los otros candidatos. Una con Beatriz Vélez en la palabra “administración”, palabra localizada en el tercer lugar de frecuencia con Silvia y en el decimonoveno con Beatriz. La otra palabra, con la cual tuvieron coincidencia todos los candidatos, fue con la palabra "capital", la cual Gaspar y Vélez tuvieron en el tercero y primer lugar respectivamente, mientras que Silvia en el vigésimo lugar.

Dentro de las palabras más usadas en los titulares sobre el candidato Antonio Gaspar, se detectaron principalmente: coalición, campaña y recorrido, siendo de índole positiva. Respecto a sus propuestas, su discurso se centró en tres palabras: cambio, alternancia y proyecto. En el caso de la candidata Beatriz Vélez, el $60 \%$ de su discurso presentado en los diarios de su campaña se fundó en palabras como: coalición, federal, mujer, acompañada, etc. Seguida de cinco 
palabras sobre el reconocimiento a la sociedad: capital, colonias, gente, comunidades y familias. Respecto a la candidata Silvia Alemán, las palabras relacionadas con sus propuestas llegaron a cinco: administración, río, Huacapa, saneamiento y resolver.

\section{Encuesta}

Para poder contrastar la información, se aplicó una encuesta a los ciudadanos de Chilpancingo que ejercieron su voto el 01 de julio del 2018, es decir, a personas mayores de 18 años y que se mantuvieron informados durante las elecciones a través de los diarios locales. Los resultados arrojados fueron los siguientes:

En la pregunta sobre popularidad de los candidatos, el $68.41 \%$ de los lectores encuestados, señalaron que Antonio Gaspar fue quien más presencia reflejó en los periódicos, en segundo lugar, con el $28.46 \%$, colocaron a Beatriz Vélez, dejando en tercer lugar a Silvia Alemán con el 3.13\%. Estos datos coinciden con la información obtenida en el análisis de contenido, ya que el candidato obtuvo el $50.3 \%$ del total de menciones en las publicaciones de los diarios durante los comicios, mientras que las otras candidatas tuvieron el $41 \%$ y el $8.7 \%$, respectivamente.

De los partidos representativos de cada candidato, la población encuestada percibe como el más popular a Morena con el $59.8 \%$, en segundo lugar, colocan al PRD con el $26.1 \%$, mientras que el $14.1 \%$ señalan al PRI como el menos posicionado.

Conforme a la aprobación que tienen los ciudadanos de cada uno de los partidos políticos, Morena obtuvo $86.4 \%$, el PRD, partido del cual proviene el candidato ganador en las elecciones, obtuvo el $41.8 \%$ y el PRI obtuvo el $30.8 \%$.

En la pregunta donde se relaciona a los candidatos con características representativas, el $65 \%$ de las personas encuestadas, relacionaron a Gaspar con palabras de índole positiva, tales como "mejores propuestas" y "trabajo". El $35 \%$ lo señaló con palabras de connotación negativa, como "no es confiable" y "corrupto".

Asimismo, el 59.3\% de los encuestados, relacionó a Beatriz Vélez con palabras de connotación negativa como "corrupción" y "no es confiable". En las palabras positivas obtuvo $40.7 \%$ con “trabajo" y "mejor partido político". En tanto, el 53.7\% relacionó a la candidata Silvia Alemán, con palabras de connotación negativa tales como "no es confiable" y "poco respaldo de su partido". Teniendo el $46.3 \%$ de palabras con tendencia positiva como "trabajo" y "busca la igualdad".

\section{Conclusiones}

El objetivo principal de este trabajo, fue analizar las características de cobertura que los diarios dieron a las campañas de los tres principales candidatos que contendieron por la presidencia municipal de Chilpancingo en las elecciones 2018. Para ello, se tuvieron también tres objetivos específicos, los cuales se cumplieron satisfactoriamente.

1. Conocer la relevancia noticiosa que se dio a la información difundida sobre cada uno de los candidatos durante el periodo de campañas 2018 por la alcaldía de Chilpancingo.

La relevancia noticiosa es la atención que prestan los medios a cierto tipo de información, la cual repercute en la agenda. Sin embargo, esto no se refiere a un papel todopoderoso de los medios, pero sí a la influencia que tienen en la formación de la opinión pública. En este caso, la relevancia se centra en la estructura (frecuencia de menciones de los candidatos, espacio/tamaño que se le dio a la información y género periodístico que se utilizó), asimismo se tomó en cuenta desde la perspectiva cognitiva, tal como la valencia/valoración, representada de tres posibles formas: positiva, negativa y neutral.

Por lo tanto, la investigación realizada permitió determinar al candidato Antonio Gaspar como el personaje con más relevancia noticiosa en los diarios locales durante esta campaña, ya que obtuvo las más altas y favorables cifras en el entorno mediático. Donde, de las 449 notas registradas, 226 fueron sobre él, lo cual representa poco más del 50\%, seguido con 184 por la candidata del PRI. Ambos fueron los que tuvieron más presencia en prensa durante la campaña, mientras que la candidata de Morena, estuvo por debajo de estos números. Cabe mencionar que fue Gaspar el único en tener una publicación de una plana completa, lo cual, sin duda, en ese periodo fue de mucha ventaja, pues se utilizó un reportaje dedicado a la presentación de propuestas 
y acercamiento con la gente. Es importante destacar que para difundir información sobre este candidato, se utilizaron todos los géneros periodísticos, es decir, hubo notas informativas, de opinión/análisis, crónica, reportaje, fotonota y entrevista, algo que sin duda, refuerza el discurso del político, al contrastar la información desde diferentes perspectivas.

Respecto a la valencia o valoración manifestada en las piezas informativas en donde se difundió información de los candidatos, la mayoría fueron positivas para el candidato que resultó ganador, teniendo en total 124, llevando casi el mismo ritmo con la candidata del PRI, Beatriz Vélez. En el caso de las notas negativas de estos dos candidatos, durante la tercera semana de campaña, surgieron a raíz de algunas diferencias entre ambos.

2 Analizar el discurso periodístico a través de los temas y titulares sobre notas publicadas de los principales candidatos a la presidencia municipal de Chilpancingo.

Al analizar los titulares y temáticas destacadas sobre el desarrollo de las campañas llevadas a cabo en Chilpancingo, se observó que las acciones políticas de cada candidato tuvieron ciertas características que formaban parte de su estrategia, algo que los diarios dieron a conocer de manera continua. Beatriz Vélez buscó el respaldo de su partido, el PRI, puesto que conocía las preferencias partidistas que el electorado de Chilpancingo había tenido hasta ese momento; se hizo acompañar por líderes y personajes representantes además de formar alianzas con otros partidos y recordar sus logros obtenidos en sus puestos anteriores. Por otro lado, Antonio Gaspar, buscó alianzas para formar una coalición, manteniendo en su discurso la presentación de propuestas, su interés por estar más cerca de la gente y sobre todo, propuso la alternancia, invitando a su campaña a quienes habían pertenecido al PRI.

Algo que sin duda afectó la imagen del PRI, fueron las declaraciones del entonces presidente municipal priista con licencia, Marco Antonio Leyva Mena, quien dejó ver un partido fragmentado y dividido en intereses, ello ocasionó un golpe irreversible a la campaña de Beatriz Vélez. Caso contrario a la candidata Silvia Alemán, quien aprovechó la visita de López Obrador para reforzar su campaña y su partido (Morena). En las últimas semanas, los candidatos siguieron con propuestas, recorridos y algunas controversias sobre temas de corrupción, popularidad y sabotaje.

3 Conocer algunas de las características percibidas por ciudadanos lectores que se mantuvieron informados con publicaciones de los diarios durante el proceso de campañas 2018.

La encuesta permitió hacer un contraste entre las publicaciones de los diarios locales y las percepciones de los votantes que se mantuvieron informaron a través de la prensa durante las elecciones 2018. Conociendo de esta manera que:

1. En cuanto a la popularidad de los candidatos, relacionado con la relevancia, se identificó una relación, puesto que las personas encuestadas reconocieron al candidato Antonio Gaspar como el que tuvo más presencia en diarios, estando más informados, durante el periodo de campañas, sobre las acciones políticas de él. Dejando en segundo lugar a la candidata del PRI, Beatriz Vélez y en tercero, a la candidata de Morena, Silvia Alemán.

2. En cuanto a popularidad del partido, relacionado con la relevancia, se observa que muchos colocaron en primer lugar a Morena, valorado positivamente con un $86 \%$, en segundo al PRD y en tercero al PRI. Lo cual nos da indicio de que la influencia del partido sobrepasaba la influencia mediática local, puesto que el fenómeno "AMLO" trascendió a lo largo del país, mostrando que la aceptación del partido era mayor a la de su candidata. Asimismo, el PRD quedó en un segundo lugar, superando en popularidad al PRI, el cual fue valorado de forma negativa por el $70 \%$ de los encuestados, quienes mencionaron que ya no se sentían identificados con este.

3. Los encuestados pudieron relacionar en un mayor porcentaje $(65 \%)$ con aspectos positivos como "mejores propuestas" y "trabajo" al candidato Antonio Gaspar, mientras que a Beatriz Vélez, casi el $60 \%$ la relacionó con aspectos negativos como "no es confiable" y "corrupta". En el caso de Silvia Alemán, destacaron de forma positiva "trabajo" y de forma negativa "no es confiable". 
Lo anterior confirma que los diarios con circulación en Chilpancingo, brindaron mayor relevancia noticiosa al candidato Antonio Gaspar Beltrán durante el proceso de campañas. Además, se elaboró un discurso periodístico favorable a la figura de este candidato, lo cual fue percibido por un gran número de ciudadanos que se mantuvieron informados a través de las publicaciones de los diarios. En el caso de Silvia Alemán Mundo, quien no tuvo mucha notoriedad en los diarios, en gran medida se respaldó de su partido, Morena, el cual la llevó a ganar el segundo lugar en la preferencia de los electores. Respecto a Beatriz Vélez, algunos mensajes tuvieron influencia en la opinión pública de manera negativa, puesto que, entre el contexto de su partido y algunos eventos no favorables, su imagen se vio afectada, quedando posicionada en el tercer lugar de los resultados electorales.

Se observó también que la aceptación del partido Morena no es coherente con la poca aceptación que tuvo su candidata, que además no tuvo mucha presencia en prensa. Mientras que la decadencia del PRI mostrada en los diarios sí va de acuerdo con los resultados de las encuestas. En tanto, Antonio Gaspar obtuvo la mayor cantidad de menciones, mientras que su partido no destacó de manera significativa. Su discurso se basó en crear una imagen política novedosa, el cual hizo llegar a un gran número de votantes al proponer un cambio para el beneficio social que llevaría a la alternancia de la capital. Además, a través de las encuestas se pudo conocer que contaba con un apoyo significativo de la ciudadanía, lo cual se vio reflejado en los resultados de las elecciones. Todo lo previamente descrito, deja en claro que la relevancia mediática favoreció a la campaña de este candidato y fortaleció su discurso.

Este trabajo muestra que las notas de prensa siguen siendo un material importante en el análisis político, ya que se documenta la relevancia brindada a ciertos asuntos seleccionados. De esta manera, se comprende el papel que juega la labor periodística en la vida y los procesos políticos. Además se evidencia la influencia que tiene la información difundida en la formación de la agenda, misma que puede percibirse por la ciudadanía. Sin embargo, la participación mediática no cuenta con una función todopoderosa, puesto que existen diversos factores que intervienen para producir efectos. Así, con estos resultados se permite visualizar el panorama actual, desde un nivel local, de los cambios políticos que acontecen en México y a la vez, abre paso a estudios más profundos en donde se desee indagar o contrastar lo aquí presentado.

\section{Referencias}

Acevedo, J. y Macassi, S. (2016). De mediadores a actores políticos. Agenda, frames y rol de los medios en el marco de la campaña electoral municipal 2014 en Lima Metropolitana. En Muñiz, C., Saldierna A. R., Morañón F. y Maldonado L. (Coord.). Estudios sobre comunicación Política en Latinoamérica (pp.167-198) México: Fontamara.

Aguayo, S. (1998). Bajo el dominio neoliberal en $\mathrm{El}$ panteón de los mitos: Estados Unidos y el nacionalismo Mexicano. México: Grijalbo.

Ascanio, A. (2014). Análisis del discurso político. México: Trillas.

Berkowitz, L., \& Rogers, K. H. (1986). A priming effect analysis of media influences. In J. Bryant, \& D. Zillman (Eds.) Perspectives on Media effects, J. Hillsdale, NJ: Lawrence Erlbaum Associates.

Campbell, A., Converse, P., Miller, W. E., \& Stokes, D. E. (1960) The American Voter. Wiley. Nueva York: Rowman \& Littlefield.

De Dios Palma, Arturo. (5 de julio, 2018). En Chilpancingo el PRI lo perdió todo. El Universal, Recuperado de http: //www.eluniversal.com.mx/elecciones-2018/en-chilpa ncingo-el-pri-lo-perdio-todo

García, V., D’Adamo, O. \& Slavinsky, G. (2005). Comunicación Política y Campañas Electorales. Barcelona: Gedisa.

Kiousis, S., \& Shields, A. (2008). "Intercandidate Agenda-Setting in Presidential Elections: Issue and Attribute Agendas in the 2004 Campaign”. Public Relations Review, vol. 34. pp. 325-330. Doi: 10.1016/j.pubrev.2008.07.004.

Martínez Pandiani, G. (2004) Homo Zapping. Política, Mentiras y Video. Buenos Aires: Ugerman.

McCombs, M. (2006). Estableciendo la agenda: El impacto de los medios en la opinión pública y en el conocimiento. Barcelona: Paidós.

McCombs, M., \& Shaw, D. (1972). The agenda-setting function of mass media, Public Opinion Quarterly, $N^{\circ} 36$, Pp. 176-187. Recuperado de : https://www.jstor.org/stab le/2747787?origin=JSTOR-pdf\&seq=1\#page_scan_t ab_contents.

Meyenberg, Y. y Lugo, J. A. (2011). Palabra y poder: Manual del discurso político. México: Grijalbo.

Muñiz, C., Saldierna A. R., Morañón F. y Maldonado L. (2016) Estudios sobre comunicación Política en Latinoamérica. México: Fontamara. 
Muñoz, A. (2003). Comunicación política en televisión y nuevos medios. Barcelona: Ariel.

Nie, N. H., Verba, S., \& Petrocik, J. R. (1976) The Changing American Voter. Cambridge, Reino Unido: University Press Cambridge.

Sabato, L. (1981) The Rise of Political Consultants. Estados Unidos: Basic Books.

Santillán, J. R. (2016). Modelo para el análisis periodístico de los temas políticos. Razón y palabra, num. 56. Recuperado de: http://www.razonypalabra.org.mx /anteriores/n59/varia/rsantillan.html.

Sweeney, W. R. (1995). The principles of planning. Boulder, Colorado: Westview Press.

Toussaint, F. (2004) Critica de la información de masas. México: Trillas.

Van Dijk, T. A. y Mendizábal, I. R. (1999). Análisis del discurso social y político. Quito, Ecuador: DocuTech: Escuela de Comunicación Social, Universidad Politécnica Salesiana. 